\title{
The recall of missing items
}

\author{
BENNET MURDOCK and DAVID SMITH \\ University of Toronto, Toronto, Ontario, Canada \\ and Defence Research and Development Canada, Toronto, Ontario, Canada
}

\begin{abstract}
In two experiments, we studied the recall of missing items. Short lists of common words were presented once and were followed immediately by a random permutation of all but one of the presented items. The task of the subject was to recall the missing item-that is, the item present in the study set but missing from the probe set. Experiment 1 replicated the high accuracy with five-item lists originally reported by Yntema and Trask (1963) and showed that the latencies were quite short (about $750 \mathrm{msec}$ ). Experiment 2 varied list length unpredictably and showed that accuracy was a function of both list length (four, five, or six items) and serial position. Latency was again quite short but was essentially independent of list length and serial position. It was possible to simulate most of the effects with the power set model with no free parameters (i.e., parameters that varied with the experimental manipulations). The results seemed to be more consistent with a direct access model (the power set model of TODAM; Murdock, 1995) than with a simple search or serial-scanning model.
\end{abstract}

A number of years ago, an interesting empirical effect was reported that is still a challenge for memory models to explain. This effect was the recall of missing items (Yntema \& Trask, 1963). Five-item lists of common monosyllabic words were presented once and were followed immediately by four of these words presented one at a time in random order. The task of the subjects was to recall the missing item - that is, the item missing from the probe set that had been presented in the study set. Thus, if the list is denoted as $\mathrm{ABCDE}$ and the probe as $\mathrm{DABE}$, the subject should recall C. Apparently, subjects had no difficulty with this task; mean percentage correct was $80 \%$. This effect is easy to demonstrate in a class or with a friend. Simply say any five common words, repeat any four of them in random order, then ask for the missing item. It generally pops out; little effort or thought seems to be involved.

In this article, we will replicate and extend this study and will compare the ability of several alternative process models to mimic the data. Why is this task (recall of missing items) worthy of further study? Like the missing-scan task (to be described below), it is a probe test and provides a method for studying storage and retrieval processes without interference from previously recalled items (output interference). Consequently, it has the potential to provide a cleaner look at underlying mnemonic processes. This is exactly what probe tests do in recognition

This research was supported by Grant APA-146 from the Natural Sciences and Engineering Research Council of Canada. We thank Matthew Duncan for some preliminary programming, Bruce Oddson for many helpful comments on the manuscript, and Trish Unruh and Wendy Sullivan for help in processing the data. Correspondence concerning this article should be addressed to B. Murdock, Department of Psychology, University of Toronto, Toronto, ON, M5S 3G3 Canada (email: murdock@psych.utoronto.ca).
(Sternberg, 1966; Waugh \& Norman, 1965), and their importance is undeniable. Probe tests have also been used in cued recall (Murdock, 1963), so the recall of missing items can be viewed as another type of probe test by which to study recall processes without output interference.

At about the same time that Yntema and Trask's (1963) study appeared, several studies of missing scan (Buschke, 1963a, 1963b) were published. Lists of digits (generally longer than those used by Yntema \& Trask, 1963) were presented, and subjects were asked to recall either all the presented items or the one missing item. Estimates of memory span were consistently greater for the missing items than for the presented items. It was concluded that the missingscan technique was a useful way of studying short-term memory storage without retrieval or output interference.

In a follow-up missing-scan study (Madsen \& Drucker, 1966), the recall of missing items was compared with the recall of presented items with pre- and postcuing of the test type. List length increased from 4 to 14 in steps of two. The researchers found that the advantage of missing over presented items decreased with postcuing (instructions as to test type presented after the list) and concluded that this advantage was likely due to different storage processes. Some time later, the case of 2 missing items was studied (Hadley, Healy, \& Murdock, 1992), and it was found to be much harder. The missing-scan test has also been used to study recall errors with small categorized sets of words (Humphreys \& Schwartz, 1971).

The recall of missing items (Yntema \& Trask, 1963) and the missing scan task (Buschke, 1963a, 1963b) differ in several ways. List length is generally longer in the missing scan task (up to 14 items or so). Also, there is a study list for the recall of missing items but no study list for the missing scan task; subjects are assumed to have a preexisting memory for the items (digits or words). Thus, the recall of missing items is basically an episodic mem- 
ory task, whereas the missing scan task assumes a preexisting ordered semantic representation.

How could the recall of missing items work? It could be some sort of serial-scanning process. Subjects could use a check-off algorithm whereby, given the probe, there were multiple scans of the probe list, where the target on each scan was one of the items from the study list. Every item in the study list that was found was tagged or checked off, and after an exhaustive set of scans, the unchecked item was recalled as the missing item. This seemed to be the idea behind Yntema and Trask's (1963) interpretation.

However, there is an alternative explanation. According to the theory of distributed associative memory (TODAM), subjects may use multiple associations (associations of several items) for the storage and retrieval of serial order information. This was originally suggested in the content-addressable distributed associative memory (CADAM) model (Liepa, 1977), a precursor to TODAM. In CADAM, it was assumed that when presented say with a five-item study list, the subjects store $\mathrm{A}$, then $\mathrm{AB}$, then $\mathrm{ABC} \ldots \mathrm{ABCDE}$, where $\mathrm{AB}$ is the association of items $\mathrm{A}$ and $\mathrm{B}, \mathrm{ABC}$ is the multiple association of A, B, and C, and so forth. For retrieval, if subjects can recall the first item, they can use $A$ to recall $B$, $\mathrm{AB}$ to recall $\mathrm{C}$, and so forth, up to $\mathrm{ABCD}$ to recall $\mathrm{E}$. For the recall of missing items, if subjects were presented with $\mathrm{ABCD}$, they could easily recall $\mathrm{E}$, the missing item.

Of course, most of the time the missing item would not be E; it would be A, B, C, or D and would be randomized from trial to trial. However, it turns out that this is not a problem. In the CADAM model, associations are based on convolution. Convolution is a way of associating two items suggested earlier as part of a convolution/ correlation formalism for the storage and retrieval of information from memory (Borsellino \& Poggio, 1973). Convolution is used for storage, and correlation is used for retrieval. Convolution can be thought of as the result of summing the negative diagonals of an outer-product matrix where the outer product in the cross-product of two item vectors. Correlation is similar, except that the positive diagonals are summed.

One can relate convolution and correlation to the multiplication and division of scalar quantities. Convolution is like multiplication, and correlation is like division. Just as algebraically abcde/dabe $=\mathrm{c}$ (and the multiplication order of both the numerator and the denominator is immaterial), so in the recall of missing items, the correlation of DABE with $\mathrm{ABCDE}$ gives $\mathrm{C}^{\prime}$, an approximation to C. A formal proof of this assertion may be found elsewhere (Murdock, 1993). Thus, for the recall of missing items, the order of presentation of the probe items would be immaterial.

TODAM has used multiple convolutions in two versions of a serial order model; the chunking model and the power set model (Murdock, 1995). In both cases, at the end of the probe presentation, the subject would have the necessary multiple associations to recall the missing item. For a list length of $L$ the subject would have both the $L$-way multiple convolution of the study set and the $(L-1)$-way convolution of the probe set in memory.

Thus, to retrieve the missing item, all the subject would have to do is correlate the $(L-1)$-way multiple convolution of the probe set with the $L$-way multiple convolution of the study set, and the missing item should pop out. The operation is independent of probe order and the position of the missing item. Consequently, we have a contrast between the serial scan method and the direct access method of a convolution-correlation model. Other distributed memory models, such as the matrix model (Humphreys, Bain, \& Pike, 1989) or the target-eventcontext-object (TECO) model (Sikström, 2000) might have to use the serial scan method because, with an outerproduct formalism, associations are not commutative. However, with a convolution-correlation formalism, associations are commutative, and this is an important difference between the two types of models (Pike, 1984).

How can we test which approach, serial scan or direct access, gives a better account of the recall-of-missingitems data? In the original Yntema and Trask (1963) article, the missing-item task was reported as an introduction to other studies. The high accuracy level was only casually mentioned in the article. Therefore, in our first experiment, we wanted to see if we could replicate their basic result. In addition, we also recorded and scored the recall latencies in order to get some idea of what sort of time scale is involved in this task.

\section{EXPERIMENT 1}

\section{Method}

Subjects. Fifteen students from the Toronto subject pool volunteered to take part in the experiment in return for course credit.

Materials. Each study set was composed of common two-syllable words randomly selected without replacement from the Toronto word pool (Friendly, Franklin, Hoffman, \& Rubin, 1982). Words were presented in black on a white background in a Times Roman font. All the stimuli were presented on a 15-in. color monitor controlled by an IBM-compatible computer.

Procedure. Each subject was given 120 study-test trials in a single experimental session. They were presented visually one at a time at a rate of one item/second on a computer monitor facing the subject. On each trial, the study set was followed by a brief pause, and then four of the five words, randomly selected, were presented one at a time in random order at the same rate (one item/second). The end of this test set was indicated by the $<>$ symbol, and the subjects were to recall the missing item.

Before the experiment began, the subjects were told exactly what would happen and were instructed to respond as quickly and accurately as possible with the missing item. They spoke into a microphone that recorded their responses for later analysis. The experiment was run without interruption or feedback about performance.

\section{Results}

Each recorded response was processed using a wave file-editing program called Cool Edit 2000. (Syntrillium Software, Scottsdale, AZ). The program allowed each recording to be viewed graphically so that it was possible to pinpoint the voice onset for the response. The software sampling rate was $22.05 \mathrm{kHz}$. We determined ac- 


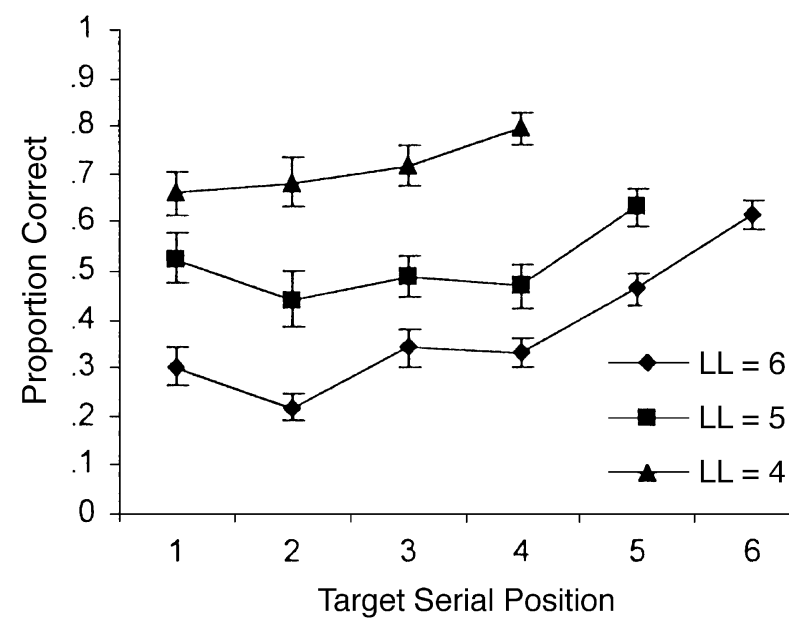

Figure 1. Proportion correct as a function of target serial position for list lengths (LLs) of four, five, and six items. The error bars represent standard errors.

curacy by listening to the recorded response and determining whether it matched the target word.

More specifically, the file-editing program allowed us to view the waveform for the subject's response and play the associated sound, starting at any point of the waveform. From the waveform, we could determine exactly when a subject made a noise. We then listened to the first noise made to determine whether it was a word. If it was a word, we noted the time for the beginning of that component of the waveform and determined whether the word was correct or an intrusion. In cases in which the first noise was not the beginning of a word, we checked the waveform at various points until the beginning of a word was found. In cases in which we could not hear a word, the trial was counted as an omission.

The mean accuracy (proportion correct) averaged over subjects and trials was .67. Although this is relatively high, it is not as high as that reported by Yntema and Trask (1963; about .80). However, their 10 subjects were laboratory staff at Lincoln Laboratory, MIT, not a random sample of undergraduate students from a large introductory psychology course. There was a small recency effect, and it was statistically significant $[F(4,56)=4.82$, $p<.01]$.

The mean latency for correct responses averaged over subjects and trials was $775 \mathrm{msec}$ This is quite short, down in the range of latencies for item recognition tasks (Sternberg, 1966). The mean latencies for Serial Positions $1-5$ were $796,783,753,745$, and $800 \mathrm{msec}$, respectively. Unlike the accuracy data, the serial position latency effect was not statistically significant $(F<1)$.

This experiment clearly replicates the general Yntema and Trask (1963) finding and, in addition, shows that the response latencies are quite short. In fact, they are so short (mean of $775 \mathrm{msec}$ ) that a serial scan seems quite improbable. These are the sort of latencies one gets in an item recognition task of the Sternberg variety (Stern- berg, 1966) that involves only a single scan. Here, it would be necessary to make four scans, and recall, rather than recognition, is required.

However, the case is not quite this simple. One must specify slopes and intercepts of a scanning function and the initiation times as well, so perhaps the argument is not compelling. More data are needed to discriminate between the models, and the models must be made much more specific if we are to get a clear-cut decision in favor of one model or the other.

As will be shown in the General Discussion section, these two approaches make different predictions about serial position and list length effects. Consequently, the second experiment was an extension of the first experiment in which list length was varied unpredictably. The reasons for looking at serial position and list length effects will be made clear in the General Discussion section, where we also will elaborate the models and apply them to our data.

\section{EXPERIMENT 2}

\section{Method}

Subjects. Twenty-two students from the same population as that in Experiment 1 volunteered to take part in the experiment in return for course credit.

Materials. The materials for this experiments were the same as those used in Experiment 1.

Design and Procedure. The procedure and methods of scoring were similar to those in Experiment 1, except that here the study lists were four, five, or six words long and, of course, the probe set was three, four, or five words long. List length was not specified in advance. The total number of lists/session was 107 . Lists with a length of four, five, and six were presented 36,35 , and 36 times so the various lengths would be about equally probable.

\section{Results}

The main results for accuracy are shown in Figures 1 and 2. For proportion correct (Figure 1), the shortest list length (four items) has the highest accuracy, whereas the longest list length (six items) has the lowest accuracy. There is a fairly clear recency effect for all three list lengths. The serial position effects were significant for lengths of five and six $[F(4,84)=2.89, p<.05$, and $F(4,105)=9.91, p<.01]$, but not for a length of four $[F(3,63)=2.58, p>.05]$.

The results for intrusions (all incorrect responses) are shown in Figure 2. They are essentially the inverse of those for proportion correct. The proportion increases with list length and decreases slightly with serial position. The picture is quite different for latency (Figure 3). As in the first experiment, the response times are quite short; more important, they are flat (for all three list lengths, $F<1$ ) and essentially independent of list length. The mean latency might be slightly shorter for a list length of four, but clearly list lengths of five and six do not differ.

A further analysis of intrusions dichotomized them into intra- and extralist intrusions. The mean latencies of both intra- and extralist intrusions were essentially flat over serial position and did not seem to vary in any sys- 


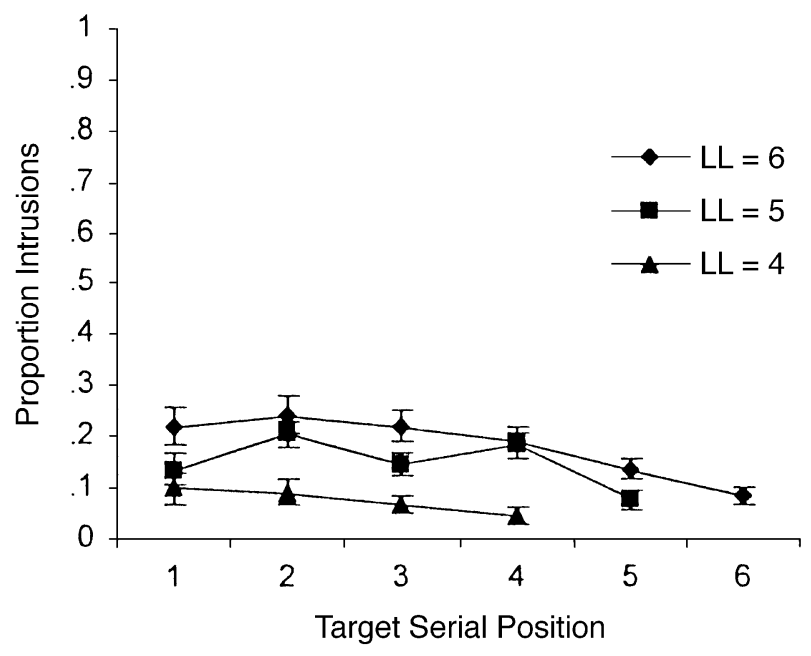

Figure 2. Proportion of intrusions as a function of target serial position for list lengths (LLs) of four, five, and six items. The error bars represent standard errors.

tematic way with list length. For frequency, the proportion of extralist intrusions did not vary with list length and was essentially constant over serial position for all list lengths. For intralist intrusions, the proportion of extralist intrusions decreased slightly over serial position, and their frequency increased with list length.

To check on the reliability of the scoring, the data from two subject files (one from each experiment) were rescored by the second author and correlated with the original scoring (one by each research assistant). For Experiment 1 the $r^{2}(119)$ value was $.999(a=-4 \mathrm{msec}$, $b=0.997)$, and for Experiment 2, the $r^{2}(107)$ value was .998 ( $a=6 \mathrm{msec}, b=0.999)$. Thus, it seems unlikely that there is a reliability problem in the scoring. We now will develop and apply the scanning and direct access models to these data.

\section{GENERAL DISCUSSION}

The fact that the latencies in both experiments were independent of list length and serial position seems to pose problems for a serial-scanning model. Of course, there are various versions of a scanning model. If the scanning is exhaustive, all that matters is list length (set size). For list lengths of four, five, and six, there will be 12,20 , and 30 or, in general, $L(L-1)$ comparisons, because all $L-1$ items in the probe set must be compared with all $L$ items in the study set. The order of the items in the probe set would be immaterial. Thus, latency curves when latency is plotted as a function of serial position should be flat, but the intercept should increase with list length.

If the scanning is self-terminating, not only list length, but also scanning direction should matter. A forward scan should yield a linear primacy effect, but a backward scan should yield a linear recency effect, when latency is plotted as a function of the serial position of the missing item. However, again the order of the items in the probe set is immaterial. That is, the number of comparisons should be exactly the same for all possible probe orders. Whether primacy or recency will be obtained depends on whether the order of scanning is forward or backward through the study set.

To see whether variability in comparison time would affect these conclusions, we ran some simulations of a scanning model. We assumed that each item in the probe set would be compared one by one with each item in the study set. The comparison time was assumed to be a normally distributed random variable $\left(t_{1}\right)$ with some mean and variance. A selection time $\left(t_{2}\right)$ also was assumed to be a normally distributed random variable. The latter was the time taken to check off the matching item in the study set and select the item in the probe set for the next comparison.

Since the presentation order of the probe set was random, the number (and hence, the total time) of each comparison varied randomly from trial to trial, as well as with list length. Rather than trying to derive analytic expressions, we simulated this serial scan with arbitrary means $(50 \mathrm{msec})$ and standard deviations $(10 \mathrm{msec})$ for $t_{1}$ (the comparison time) and $t_{2}$ (the selection time).

In the simulations, on each trial, a list of $L-1$ integers was put in random order, and then a serial self-terminating scan was carried out. The fact that we used integers in the simulations, rather than words, was only for convenience. We did not make use of their scale properties. The first item in this probe (i.e., the first item in this randomized list) was compared item by item with the integers $1,2, \ldots, L$, and the time to make this comparison was a random variable that was a random sample from the $t_{1}$ distribution. If no match was found, the process continued (with an additional time that was a random sample from the $t_{2}$ distribution) until a match was found. The total time for that trial was assumed to be the sum of all the comparison and selection times. There were 100

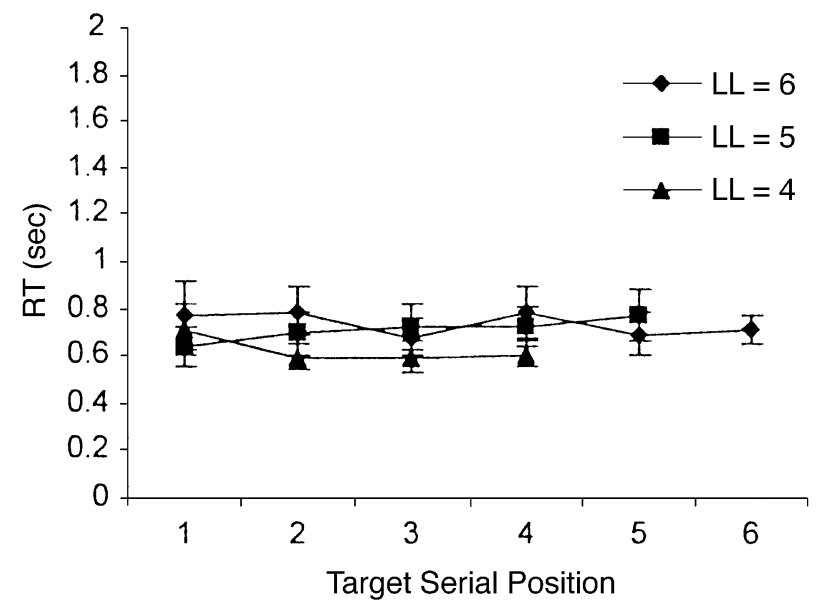

Figure 3. Reaction time (RT) as a function of target serial position for list lengths (LLs) of four, five, and six. The error bars represent standard errors. 
replications for each length (four, five, and six) and each possible missing-item serial position, and we then computed means and standard deviations for each serial position at each list length.

The results showed that adding variability did not change the predictions, and the variability seemed to be a linear function of the means. Thus, this particular version of a serial-scanning model seems to fail on all counts, and even adding variability does not seem to help. We also repeated the simulation with the log-normal distribution, which has been shown to fit recognition latencies about as well as the ex-Gaussian (Ratcliff \& Murdock, 1976), and the results were essentially the same as those with normally distributed strength distributions.

However, it should be made clear that the arguments above assume that the retrieval process starts with the end of the presentation of the probe. It is, of course, possible that some study items are checked off or tagged during the presentation of the probe set. Even so, such a model would still probably have to predict a small serial position effect, but it might be so small as to be unnoticeable. In any event, the tagging mechanism would have to be specified and detailed predictions developed before it could be evaluated.

\section{Direct Access}

To compare a TODAM direct access view with a serial scan model, we simulated that as well. To relate this to previous work, we used a version of TODAM that includes multiple convolutions. We could have used the chunking model or the power set model, but for reasons described elsewhere (Murdock, 2005), we used the power set model. The main reason is the complexity of the chunking model; higher order convolutions would be necessary, and they seemed unlikely here.

The power set of a set of single items is all single items and all combinations of these single items. If there were four items (a, b, c, and d), the power set would be $\{a, b$, c, d, ab, ac, ad, bc, bd, cd, abc, abd, acd, bcd, abcd $\}$. Thus, we have the single items (a, b, c, and d), all pairwise combinations ( $a b, b c, c d, a c, a d$, and bd), all threeway combinations (abc, abd, acd, and bcd), and the one four-way combination (abcd). ${ }^{1}$

According to the power set model, if $\mathbf{f}_{j}$ is the $j$ th item in a list of $L$ items, $\mathbf{Q}$ is the short-term memory system (Murdock, 1983, Figure 2, p. 321), $\delta$ is a start vector, and a $\operatorname{star}(*)$ denotes convolution, the storage equation is

$$
\mathbf{Q}_{j}=\mathbf{Q}_{j-1}+\mathbf{f}_{j}+\mathbf{f}_{j} * \mathbf{Q}_{j-1}, \mathbf{Q}_{1}=\mathbf{f}_{1}+\delta * \mathbf{f}_{1} .
$$

This storage equation automatically generates the power set of $L$ items. $^{2}$

For the recall of missing items, all we need is the $L$ way combination for the study set and the $(L-1)$-way combination for the probe set. Of course, we know the model can do more than recall missing items. Using the single items ( $a, b, c$, and $d)$, it can do item recognition, and using the adjacent pairwise combinations ( $\mathrm{ab}, \mathrm{bc}, \mathrm{ad}$, and cd), it can do sequential probe tests. Unless a derivedlists paradigm is used (Slamecka, 1985), the rest is noise.

However, noise is important theoretically. For the recall of missing items, without normalization the power set model automatically predicts an increase in noise as $L$ increases. Speaking generally, an increase in noise leads to a decrease in accuracy, and that is exactly what we find. But what exactly is noise?

In TODAM, as in the matched-filter model (Anderson, 1973), items are represented by random vectorsthat is, vectors whose elements are random variables. These random variables are random samples from a normal distribution with a mean of zero and a variance of $1 / N$, where $N$ is the dimensionality of (or number of features in) the item vector. The multiple convolution of any $L$ items is also a vector, so naturally the multiple convolution of $L-1$ items is also a vector. In previous applications of TODAM, we have used linear convolution. Here, we use circular convolution, the product of the Fourier transform of the item vectors. The advantages are that there is no increase in dimensionality as $L$ increases and, by using the fast Fourier transform, the simulations run much faster. The disadvantage is a higher noise level. An extensive analysis of circular convolution and an application to frames in discourse processing may be found elsewhere (Plate, 1995).

For retrieval, the $(L-1)$ component probe vector is correlated with (divides) the $L$-item component study vector, and the result is an approximation to the missing item. The goodness of approximation of the retrieved item $\mathbf{f}^{\prime}$ to the prime target (missing) item $\mathbf{f}_{k}$ can be assessed by the dot product $\mathbf{f}_{k}^{\prime} \cdot \mathbf{f}_{k}$. The dot product is simply the sum of the products of the corresponding elements in $\mathbf{f}_{k}^{\prime}$ and $\mathbf{f}_{k}$.

The expected value $(E)$ of this dot product is simply the mean resemblance of the retrieval item to the target item, but of course, it also has a variance. The variance is noise. Consequently, we can compute a signal-to-noise $(\mathrm{S} / \mathrm{N})$ measure for each serial position, and this gives us a way to predict accuracy at each serial position. The $\mathrm{S} / \mathrm{N}$ ratio at each serial position for each list length is simply the dot product mean divided by the dot product standard deviation. The $\mathrm{S} / \mathrm{N}$ ratio is the reciprocal of the coefficient of variation and comes from the informationprocessing field (Parzen, 1960). The dot product is the dot product of the retrieved item approximation $\left(\mathbf{f}_{k}^{\prime}\right)$ with the target item $\left(\mathbf{f}_{k}\right)$, where the approximation is obtained by correlating (dividing) the study vector by the probe vector. Both the study and the probe vectors are constructed by Equation 1, using TODAM item vectors of some arbitrary dimensionality $(N)$.

What about latency? There are two ways to look at latency. According to the first way, latency should be independent of both list length and serial position, which of course, it is (Figure 3). Why is this so? Because a study item or a probe item vector is an item vector regardless of the number of item vectors used for each. Correlation (di- 
vision) is a single operation, and if you divide two sums of normally distributed zero-centered random variables, clearly the number of variables entering into each sum should be immaterial. This could be checked by computer simulation, but it hardly seems to be necessary.

However, according to the second way, latency should be affected by list length and, possibly, by the serial position of the missing item as well. As for list length, perhaps encoding lags item presentation and the lag increases with serial position. The dot product cannot be computed until encoding is complete, so even though computing the dot product is not affected by list length, the response time must include variable lags as well. Thus, by this view, latency should increase with list length, but how much and in what way is not clear.

Given that these two alternatives were not specified in advance, we obviously cannot claim that TODAM predicted the latency data of our experiment. What we can say is that the result is perfectly consistent with the first TODAM alternative. Therefore, as a better test of this direct-access view, we need to consider the accuracy predictions, and as with the serial scan approach, that requires simulations.

\section{Power-Set Simulation}

The purpose of the simulation was to get the means and variances of the resemblance of the retrieved item with the target item that was the missing item. On each trial, after a random set of $L$-dimension $N$ items had been constructed, the memory vector was constructed by the power set model storage equation (Equation 1). Then a random $L-1$ of these items were selected, randomly ordered, and the probe vector was constructed in the same way, except that the start vector $(\delta)$ was not used, since recall was not required.

After the probe and study vectors had been constructed, the former was correlated with the latter to obtain the retrieved item. Circular convolution was used throughout, and for correlation, we convolved the involution of the probe vector with the memory vector. This has been shown to be identical with convolution (Schonemann, 1987). With circular convolution, the first element in the probe item stays fixed, but all the subsequent items are mirror reversed. The resemblance was assessed in two ways, both by the dot product of the retrieved item with the target item and by the cosine of the angle between them. It has been argued that the cosine (which is essentially a normalized dot product) is a better measure of resemblance, because it corrects for unequal lengths of the two vectors (Goebel \& Lewandowsky, 1991). Because the cosine seems more appropriate for the power set model we will report only the cosine results.

Using the cosine measure, we computed means and variances for each missing item serial position pooled over replications. On each trial, the serial position of the missing item was randomly determined, and all this was done separately for list lengths $(L)$ of four, five, and six. The value of $N$ was set to $1,024\left(2^{10}\right)$, and there were
10,000 replications for each list length. The entire simulation, using Mathematica 4.0, took a little more than $24 \mathrm{~h}$ on a Pentium III computer.

The results of the simulation are shown in Table 1 . The results are $\mathrm{S} / \mathrm{N}$ ratios; as has been noted, the $\mathrm{S} / \mathrm{N}$ ratios are the means over the standard deviations. The magnitudes are not important (they can easily be transformed into a forced-choice probability correct measure); what is important are the patterns. The diagonals of each matrix correspond to the resemblances of the missing item, and the off-diagonal elements correspond to the resemblances of all possible intralist intrusions.

There are three results of interest. First, the off-diagonal values are reasonably symmetric for all three list lengths. This implies that earlier and later intrusions should be about equally likely, and that is essentially what we found in the experiment. However, there were not enough intrusions for a statistical analysis, so little weight should be put on this correspondence. Second, the diagonal means clearly decrease in magnitude, going from a list length of four to a list length of six. This is exactly the pattern we found for the accuracy results (Figure 1). Finally, if anything, there is a slight primacy effect; the resemblance values seem to decrease monotonically with serial position for all three list lengths. This result is not what the experiments showed; they showed recency, not primacy (Figure 1). How can this be explained?

Suppose, for whatever reason, that the study items in the list were graded monotonically, either from strongest to weakest or from weakest to strongest. A few simulations showed quite clearly that the resemblance $\mathrm{S} / \mathrm{N}$ values were aligned accordingly. That is, when there was a primacy strength gradient for the items, the $\mathrm{S} / \mathrm{N}$ values also showed a primacy gradient, whereas when there was a recency strength gradient, the $\mathrm{S} / \mathrm{N}$ values also showed a recency gradient.

Although it does not follow that the resemblance $\mathrm{S} / \mathrm{N}$ gradients we found in Experiment 2 were due to recency, if there were recency in the item strengths and this were incorporated into the power set model simulations, we would presumably get the right resemblance serial position results in the simulation. Although recency in serial recall seems dead wrong, there is the neglected factor of output interference, which does in fact suggest a recency component to strength (Cowan, Saults, Elliott, \& Moreno, 2002). Probe tests in serial recall almost invariably show recency and not primacy (e.g., Murdock, 1968).

It should be made clear that there were no free parameters in these simulations. The only parameter was the TODAM $N$ (number of features), which was arbitrarily set to 1,024 . Thus, the fact that the simulation generated the right pattern of results in even two of the three measures is some consolation. Although our explanation of the discrepancy in the serial position effects is somewhat speculative, the point is that the incorrect result in the power set simulation is not necessarily fatal. By any context drift notion (Estes, 1955), context should surely show a recency effect. Consequently, if context were in- 
Table 1

Resemblance Signal-to-Noise Ratios From Simulations of the Power-Set Model for List Lengths of 4, 5, and 6

\begin{tabular}{|c|c|c|c|c|c|}
\hline & 4.79 & 1.48 & 1.48 & 1.46 & \\
\hline & 1.84 & 4.32 & 1.81 & 1.83 & \\
\hline & 1.80 & 1.79 & 4.21 & 1.80 & \\
\hline & 1.86 & 1.82 & 1.78 & 4.21 & \\
\hline \multicolumn{2}{|c|}{3.61} & 1.26 & 1.31 & 1.27 & 1.29 \\
\hline \multicolumn{2}{|c|}{1.63} & 3.42 & 1.51 & 1.55 & 1.55 \\
\hline \multicolumn{2}{|c|}{1.66} & 1.65 & 3.53 & 1.61 & 1.62 \\
\hline \multicolumn{2}{|c|}{1.61} & 1.57 & 1.57 & 3.40 & 1.57 \\
\hline \multicolumn{2}{|c|}{1.64} & 1.56 & 1.57 & 1.57 & 3.45 \\
\hline 2.71 & 0.95 & 0.96 & 1.03 & 0.99 & 1.00 \\
\hline 1.32 & 2.69 & 1.26 & 1.25 & 1.26 & 1.32 \\
\hline 1.34 & 1.27 & 2.70 & 1.29 & 1.30 & 1.30 \\
\hline 1.31 & 1.24 & 1.30 & 2.73 & 1.32 & 1.28 \\
\hline 1.29 & 1.26 & 1.26 & 1.19 & 2.62 & 1.20 \\
\hline 1.38 & 1.29 & 1.29 & 1.29 & 1.26 & 2.74 \\
\hline
\end{tabular}

Note-For each array the rows present the serial positions of the missing items and the columns present the resemblance values (as measured by the cosine) of the retrieved item to each of the possible list items. The number of features in the item vectors (TODAM $N$ ) was 1,024 and there were 10,000 replications for each set size (list length).

cluded in TODAM, the primacy might change to recency. However, this possibility needs to be developed and tested.

Another interpretation might be in terms of directed forgetting (Bjork, LaBerge, \& Legrand, 1968; MacLeod, 1998). Perhaps, as each probe item is presented, it acts as a to-be-forgotten cue, so the corresponding item is tagged or deleted from the memory set. Then at the end of the probe set presentation, the subject recalls the one "remaining" (i.e., not forgotten) item. This view would seem to suggest that probe order should matter. Thus, if the probe order for study set $\mathrm{ABCDE}$ was $\mathrm{ABCD}$, the recall of $E$ would be easiest, but if the probe order were EDCB, recall of A would be hardest (at least, most error prone), because of all the processing that would be required during the presentation of the probe set. However, an analysis of probe order showed that, in fact, probe order did not matter; even these two extreme cases (ABCD for $\mathrm{E}$ and $\mathrm{EDCB}$ for $\mathrm{A}$ ) did not differ. ${ }^{3}$

More specifically, if the representation of each item in the study set was tagged when the corresponding item in the probe set was presented, we would be back to a scanning model. If the probe order matched the order of the study items for a list length of $L, L$ scans would be necessary to find the missing item, but if it were in the reverse order, then $L$ ! ( $L$ factorial) scans would be necessary to find the missing item. These are the best and worst cases ( $L$ scans and $L$ ! scans, respectively), and intermediate cases would be ordered accordingly. Overall performance should be affected by probe order, and as has been noted, this was not the case. Of course this is only one possible version of a directed-forgetting interpretation; other versions might fare better.
Directed forgetting is like a scan-and-drop strategy. If there were directed forgetting, you would have to find each item to "forget" it. It seems reasonable to assume that if the scan were in the forward direction, there would be fewer errors if the probe order was $A B C D$ than if it was EDCB, because you would have to scan 4 items in the former case to forget everything but $\mathrm{E}$, whereas you would have to scan 10 items (ABCD, $\mathrm{ABC}, \mathrm{AB}$, and $\mathrm{A}$ ) to end up with $\mathrm{A}$. The same argument applies the other way if the scan to forget goes in the backward direction through the memory set and, as has been noted, the two orders do not differ.

\section{Other Models}

The classic issue in the serial order area is the issue of item-based versus position-based associations (Young, 1968). Are the associations underlying the storage and retrieval of items some form of item-to-item associations or some form of item-to-position associations? Current models of serial order are divided on this issue; some models, such as the bind-cue-decide model (Dennis \& Humphreys, 2001), the serial-order-in-a-box (SOB) model (Farrell \& Lewandowsky, 2002), the feature model (Nairne, 1990), the primacy model (Page \& Norris, 1998), and the chunking and power set models of TODAM (Murdock, 1995), are all basically item based, whereas the dual-trace array perturbation model (Estes, 1997), the start-end model (Henson, 1998), the network model (Burgess \& Hitch, 1999), and the oscillator-based associative recall model (Brown, Preece, \& Hulme, 2000) may be considered position-based models (Howard \& Kahana, 2002).

The results of the present study would seem to pose problems for almost all of these models. Not only can subjects recall missing items with a fairly high degree of accuracy, but also the latency averages are quite short. Also, within the range studied, latency is unaffected by list length and serial position of the missing item, whereas accuracy is affected by both. Accuracy decreases with list length, and in addition, there is a fairly clear monotonic recency effect for the three lengths we studied.

Why are these results problematic? It seems likely that any position-based model would have to resort to some sort of a serial-scanning model to explain how recall of missing items is carried out, and the serial-scanning model we simulated showed that this is wrong on almost every count. Of course, one could assume that the comparison and selection times are vanishingly small, but then such a version of a scanning model becomes equivalent to a direct access model. Also, how then could such positionbased models account for latency data in other tasks?

Most item-based models would also have to posit a serial scan (i.e., a serial scan with check-off). How else could the task be performed? Assuming this is the case, our results also raise problems for most item-based models. A possible exception might be the SOB model. Perhaps it could use antilearning (subtract the autoassociations of the items in the probe set from the autoassociations of the items in the memory set), and the re- 
sult would be the missing item itself. Furthermore, it would probably predict the greater difficulty for the missing-two study (Hadley et al., 1992). However, for accuracy, it would probably predict primacy, not recency, because of the attenuation process used to explain serial position effects in serial recall.

\section{Conclusion}

Our study clearly replicates the original Yntema and Trask (1963) study in that we found, as did they, that accuracy for the recall of missing items with short lists of common words is quite high. Furthermore, the latencies are surprisingly short-roughly, $750 \mathrm{msec}$ in both experiments. Although there is both a serial position and a list length effect for accuracy, there is neither for latency (Kahana \& Loftus, 1999).

The data seem to argue against the serial scan suggestion of Yntema and Trask (1963). More generally, they rule out what seems to us to be the most reasonable version of a serial search model, and a direct access model using the multiple convolutions of CADAM and TODAM seems to be closer to the truth. However, even this direct access view is not consistent with the serial position effects for accuracy, but perhaps this discrepancy could be resolved by including context effects.

We would like to make it clear that we are not rejecting all scanning models. As one reviewer pointed out, we have discredited one particular scanning model: one with "a linear ordered list representation that is processed at the time of the test cue . . with no preprocessing (e.g., some form of tagging), with an exhaustive processing rule, independence in rates of processing individual items, an assumption of unlimited capacity at the level of the item processing (i.e., same amount of time available, stochastically, for each item), an assumption of limited capacity at the level of the list (i.e., an expectation for longer times with longer lists), and (possibly) a starting rule that specifies either the beginning or the end of the ordered list as the invariant starting position for the scan."

The other reviewer made the same point and suggested some alternatives that might work-namely, "(a) the possibility that the search set starts while the probe set is being presented; (b) the possibility that only some of the items from the probe set are considered; and (c) the possibility that the subject compares each item in the target set with the probe set (both sets are in memory so I do not see why one must assume that probe items are compared with target items as opposed to vice versa)." We have assumed that the retrieval process does not start until the end of the presentation of the probe set, and we admit that we cannot reject the possibilities suggested by either reviewer. However, it would be necessary to specify the details of these alternative possibilities and show how they could do at least as good a job as TODAM.

This same reviewer also said that, "In my opinion, this is the first finding which is truly suggestive of a direct access account," and this may be the main contribution of the present article. The search metaphor has long enjoyed great popularity as the way information is retrieved from memory, but perhaps it is time to take the direct access notion seriously. TODAM has always assumed a direct access retrieval process from a distributed memory system; other distributed memory and connectionist models do likewise, so we feel that this possibility deserves more study.

\section{REFERENCES}

Anderson, J. A. (1973). A theory for the recognition of items from short memorized lists. Psychological Review, 80, 417-438.

Bjork, R. A., LaBerge, D., \& Legrand, R. (1968). The modification of short-term memory through instructions to forget. Psychonomic Science, 10, 55-56.

Borsellino, A., \& Poggio, T. (1973). Convolution and correlation algebras. Kybernetik, 122, 113-122.

Brown, G. D. A., Preece, T., \& Hulme, C. (2000). Oscillator-based memory for serial order. Psychological Review, 107, 127-181.

Burgess, N., \& Hitch, G. J. (1999). Memory for serial order: A network model of the phonological loop and its timing. Psychological Review, 106, 551-581.

BuschKe, H. (1963a). Relative retention in immediate memory determined by the missing scan method. Nature, 200, 1129-1130.

BusCHKE, H. (1963b). Retention in immediate memory estimated without retrieval. Science, 140, 56-57.

Cowan, N., Saults, J. S., Elliott, E. M., \& Moreno, M. V. (2002). Deconfounding serial recall. Journal of Memory \& Language, 46, 153-177.

Dennis, S., \& Humphreys, M. S. (2001). A context noise model of episodic word recognition. Psychological Review, 108, 452-478.

EsTES, W. K. (1955). Statistical theory of spontaneous recovery and regression. Psychological Review, 62, 145-154.

Estes, W. K. (1997). Processes of memory loss, recovery, and distortion. Psychological Review, 104, 148-169.

FARRELL, S., \& LEWANDOWSKY, S. (2002). An endogenous distributed model of ordering in serial recall. Psychonomic Bulletin \& Review, 9, 59-79.

Friendly, M., Franklin, P. E., Hoffman, D., \& \& Rubin, D. C. (1982). The Toronto Word Pool: Norms for imagery, concreteness, ortographic variables, and grammatical usage for 1,080 words. Behavior Research Methods \& Instrumentation, 14, 375-399.

Goebel, R. P., \& LeWANDowsky, S. (1991). Retrieval measures in distributed memory models. In W. E. Hockley \& S. Lewandowsky (Eds.), Relating theory and data: Essays on human memory in honor of Bennet B. Murdock (pp. 509-527). Hillsdale, NJ: Erlbaum.

Hadley, J. A., Healy, A. F., \& Murdock, B. B. (1992). Output and retrieval interference in the missing-number task. Memory \& Cognition, 20, 69-82.

Henson, R. N. A. (1998). Short-term memory for serial order: The start-end model. Cognitive Psychology, 36, 73-137.

HowARD, M. W., \& KaHANA, M. J. (2002). A distributed representation of temporal context. Journal of Mathematical Psychology, 46, 269-299.

Humphreys, M. S., Bain, J. D., \& Pike, R. (1989). Different ways to cue a coherent memory system: A theory for episodic, semantic, and procedural tasks. Psychological Review, 96, 208-233.

HUMPHREYS, M. S., \& SCHWARTZ, R. M. (1971). Within category generalization in a missing scan paradigm. Journal of Verbal Learning \& Verbal Behavior, 10, 694-701.

Kahana, M. J., \& Loftus, G. (1999). Response time versus accuracy in human memory. In R. J. Sternberg (Ed.), The nature of cognition (pp. 323-384). Cambridge, MA: MIT Press.

LIEPA, P. (1977). Models of content addressable distributed associative memory $(C A D A M)$. Unpublished manuscript, University of Toronto.

MACLEOD, C. M. (1998). Directed forgetting. In J. M. Golding \& C. M. MacLeod (Eds.), Intentional forgetting: Interdisciplinary approaches (pp. 1-57). Mahwah, NJ: Erlbaum.

MADSEN, M. C., \& DRUCKER, J. M. (1966). Immediate memory by missing scan and modified digit span. Psychonomic Science, 6, 283-284 MuRDOCK, B. B. (1963). Short-term retention of single paired associates. Journal of Experimental Psychology, 65, 433-443. 
Murdock, B. B. (1968). Serial order effects in short-term memory. Journal of Experimental Psychology, 76(4, Pt. 2), 1-15.

Murdock, B. B. (1983). A distributed memory model for serial-order information. Psychological Review, 90, 316-338.

Murdock, B. B. (1993). Derivations for the chunking model. Journal of Mathematical Psychology, 37, 421-445.

Murdock, B. B. (1995). Developing TODAM: Three models for serialorder information. Memory \& Cognition, 23, 631-645.

Murdock, B. B. (2005). Storage and retrieval of serial-order information. Memory, 13, 259-266.

NAIRNE, J. S. (1990). A feature model of immediate memory. Memory \& Cognition, 18, 251-269.

Page, M. P. A., \& Norris, D. G. (1998). The primacy model: A new model of immediate serial recall. Psychological Review, 105, 761-781.

PARZEN, E. (1960). Modern probability theory and its applications. New York: Wiley.

PIKE, R. (1984). Comparison of convolution and matrix distributed memory systems for associative recall and recognition. Psychological Review, 91, 281-294.

Plate, T. A. (1995). Holographic reduced representations. IEEE Transactions on Neural Networks, 6, 623-641.

RATCLIFF, R., \& Murdock, B. B. (1976). Retrieval processes in recognition memory. Psychological Review, 83, 190-214.

Schonemann, P. H. (1987). Some algebraic relations between involutions, convolutions, and correlations with applications to holographic memories. Biological Cybernetiks, 56, 367-374.
SiKström, S. (2000). The TECO theory and lawful dependency in successive episodic tests. Quarterly Journal of Experimental Psychology, 53A, 693-728.

SlamecKa, N. J. (1985). Ebbinghaus: Some associations. Journal of Experimental Psychology: Learning, Memory, \& Cognition, 11, 414-435. STERNBERG, S. (1966). High-speed scanning in human memory. Science, 153, 652-654.

Waugh, N. C., \& Norman, D. A. (1965). Primary memory. Psychological Review, 72, 89-104.

YnTEMA, D. B., \& Trask, F. P. (1963). Recall as a search process. Journal of Verbal Learning \& Verbal Behavior, 2, 65-74.

Young, R. K. (1968). Serial learning. In T. R. Dixon \& D. L. Horton (Eds.), Verbal behavior and general behavior theory (pp. 122-148) Englewood Cliffs, NJ: Prentice-Hall.

\section{NOTES}

1. Technically, the power set also should include the null element, but that does not seem appropriate here.

2. The reason why $\mathbf{Q}_{1}=\mathbf{f}_{1}+\delta * \mathbf{f}_{1}$ is to solve a problem with the original version of the power set model (Murdock, 1995).

3. This analysis was done independently by Bruce Oddson, and we thank him for his interest.

(Manuscript received February 12, 2003; revision accepted for publication June 28, 2004.) 